\title{
Blended learning: panorama y perspectivas
}

\section{Blended learning: overview and expectations}

\author{
Antonio Bartolomé-Pina \\ Universidad de Barcelona \\ Rosa García-Ruiz \\ Universidad de Cantabria \\ Ignacio Aguaded \\ Universidad de Huelva
}

\section{Resumen}

La enseñanza se ha transformado, al comienzo del nuevo siglo, con las tecnologías de la comunicación. Los modelos de enseñanza-aprendizaje mediados por computadoras han supuesto un revulsivo para nuevas formas de enseñar y aprender más allá de las coordenadas espacio-temporales. E-Learning, blended learning y mobile learning ofrecen un nuevo universo de interactividad para la relación didáctica. En este trabajo, se ofrece una revisión del estado de las investigaciones y la literatura científica sobre esta nueva realidad que supone el blended learning, analizando en las principales bases de datos científicas su conceptualización con más de 7.000 registros en Web of Science y casi 95.00o en Google Scholar. Los resultados del trabajo demuestran el fuerte impulso de las buenas prácticas y la consecuente investigación en todas las áreas y niveles educativos, competencias transversales, metodologías docentes y tecnologías y materiales educativos, concluyéndose que las nuevas formas de enseñar y aprender han de ser estudiadas y comprendidas en sus interacciones con los nuevos medios y contextos de aprendizaje, a partir de la irrupción de la revolución del blended learning.

Palabras clave: enseñanza-aprendizaje; innovación; TIC; interacción, blended learning; revolución educativa.

\begin{abstract}
At the start of the new century, teaching was transformed by the new communication technologies. Teaching-learning models, mediated through computers, have catalyzed new ways of teaching and learning beyond spatial and temporal coordinates. E-learning, blended learning and mobile learning offer a new universe of interactivity for teaching purposes. This work reviews the state of research studies and scientific literature on the new reality created by blended learning, analyzing its conceptualizations in the main scientific databases, with more than 7000 entries in Web of Sciences and almost 95000 in Google Scholar. The outcomes of this work show the strong momentum of good practices and the subsequent research in all the areas and levels of education, transversal competencies, teaching methods, educational
\end{abstract}


materials and technologies. The main conclusion is that new ways of teaching and learning should be studied and understood regarding their connections with new learning media and contexts, starting from the incursion of the blended learning revolution.

Keywords: teaching-learning; innovation; ICT; interaction, blended learning; educational revolution.

\section{¿UN NUEVO TÉRMINO O UN NUEVO CONCEPTO?}

La irrupción de las tecnologías de la comunicación en la enseñanza ha supuesto una auténtica revolución en todos los ámbitos de la vida, pero especialmente en el mundo educativo (Rubia y Guitert, 2014). Con el nuevo milenio, se abrió paso un nuevo término en la literatura pedagógica: el blended learning, después de los diseños de eLearning, quizás como consecuencia de ellos, y antes de que apareciera el término mobile learning (figura 1). Este orden, en el tiempo, se mantuvo inicialmente en lo que respecta a su importancia en el sistema educativo. Pero ¿en qué se ha convertido y qué se entiende hoy por blended learning? ¿Sigue siendo un tema relevante para los educadores?

Figura 1. Evolución de los términos "blended learning”, "eLearning” y "mobile learning” en la literatura científica inglesa (1995-2008)

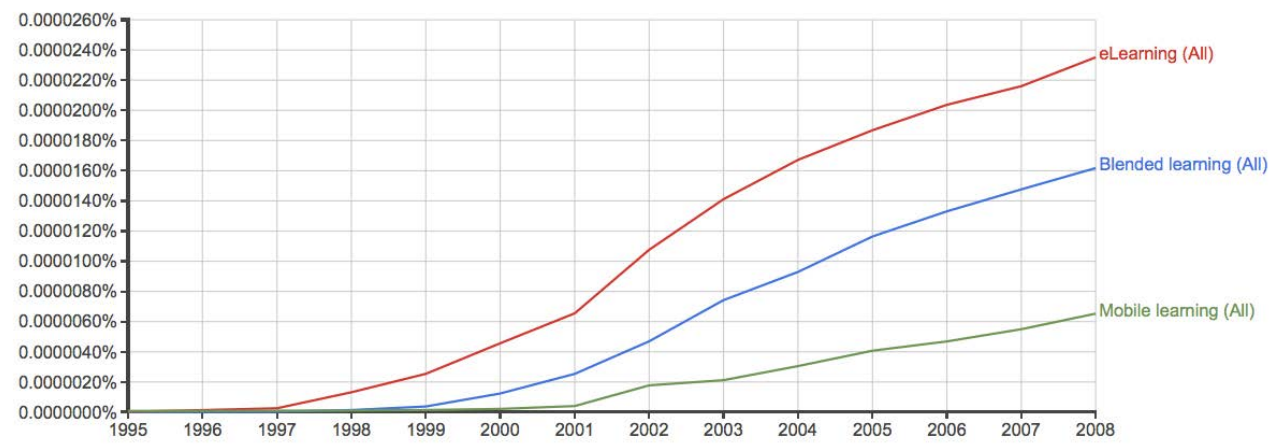

Casi dos décadas han transcurrido desde que Internet ha dado un salto cualitativo para entender un nuevo modelo educativo, si bien el mobile learning no se generaliza hasta la segunda década del siglo con la revolución de los smartphones y su uso masivo por la población, básicamente en actividades de ocio y poco formales desde un punto de vista educativo. Las tendencias no paran de crecer y a los nuevos dispositivos, cada vez más accesibles y ubicuos, se unen nuevas tendencias tecnológicas y estrategias didácticas como la realidad aumentada y la flipped classroom. Estos son modelos de 
instrucción dinámicos, no estáticos, y están evolucionando bruscamente en múltiples sentidos con el desarrollo de innovaciones en las aulas, avaladas por los avances de la tecnología. No sabemos aún con precisión si se trata de meros tanteos, pero es probable que nos encontremos ya a las puertas de una auténtica transformación de los modelos clásicos. Los avances tecnológicos, la popularización de los dispositivos, los múltiples accesos a la información y pronto el "Internet de las cosas" y, sobre todo, desde el punto de vista didáctico, los nuevos modos de enseñar y aprender pueden generar confluencia hacia un movimiento global educativo, conectado con la constante expansión de la demanda global de ciudadanos más formados y de profesionales más capacitados.

\section{CONCEPTUALIZACIÓN}

Blended learning es ya hoy un término de uso frecuente y habitual en los manuales de medios y las tecnologías en la enseñanza (Aguaded y Cabero, 2013). Fue definido como "el uso mezclado de entornos presenciales y no presenciales" (Bartolomé, 2004), pero ha evolucionado hacia diseños pedagógicos en los que se precisa el tipo de actividades y la relación entre ambos entornos, por ejemplo, el flipped classroom. Como resultado, hoy los entornos de blended learning (BL) presentan gran variedad de formatos y diseños pedagógicos. Margulieux, McCracken y Catrambone (2016) clasificaron los cursos BL utilizando estas cuatro categorías:

- Localización: en casa, en un lugar público o en un lugar específico.

- Medio de distribución (si hay distribución de materiales).

- Tipo de instrucción: magistral, activo, etc.

- Sincronía: actividades simultáneas, sucesivas en la línea de tiempo.

Este conjunto de variables ofrece un modelo complejo que recoge la variedad de términos que se utilizan para describir en inglés los sistemas BL con cierta coherencia. En español también se han utilizado diferentes términos para referirse a esta forma de combinar enseñanza presencial y virtual: blended learning, enseñanza semipresencial, aprendizaje mixto o aprendizaje mezclado (Salinas, 1997).

Un estudio del número de veces que aparecen los diferentes términos en la literatura académica a partir de los resultados de Google Scholar muestra cómo el término inglés blended learning ha terminado por imponerse en la literatura en castellano (figura 2). Como consecuencia, en este artículo utilizaremos genéricamente la expresión inglesa blended learning (de modo abreviado: BL). 
Figura 2. Media anual de aparición del término blended learning en artículos científicos

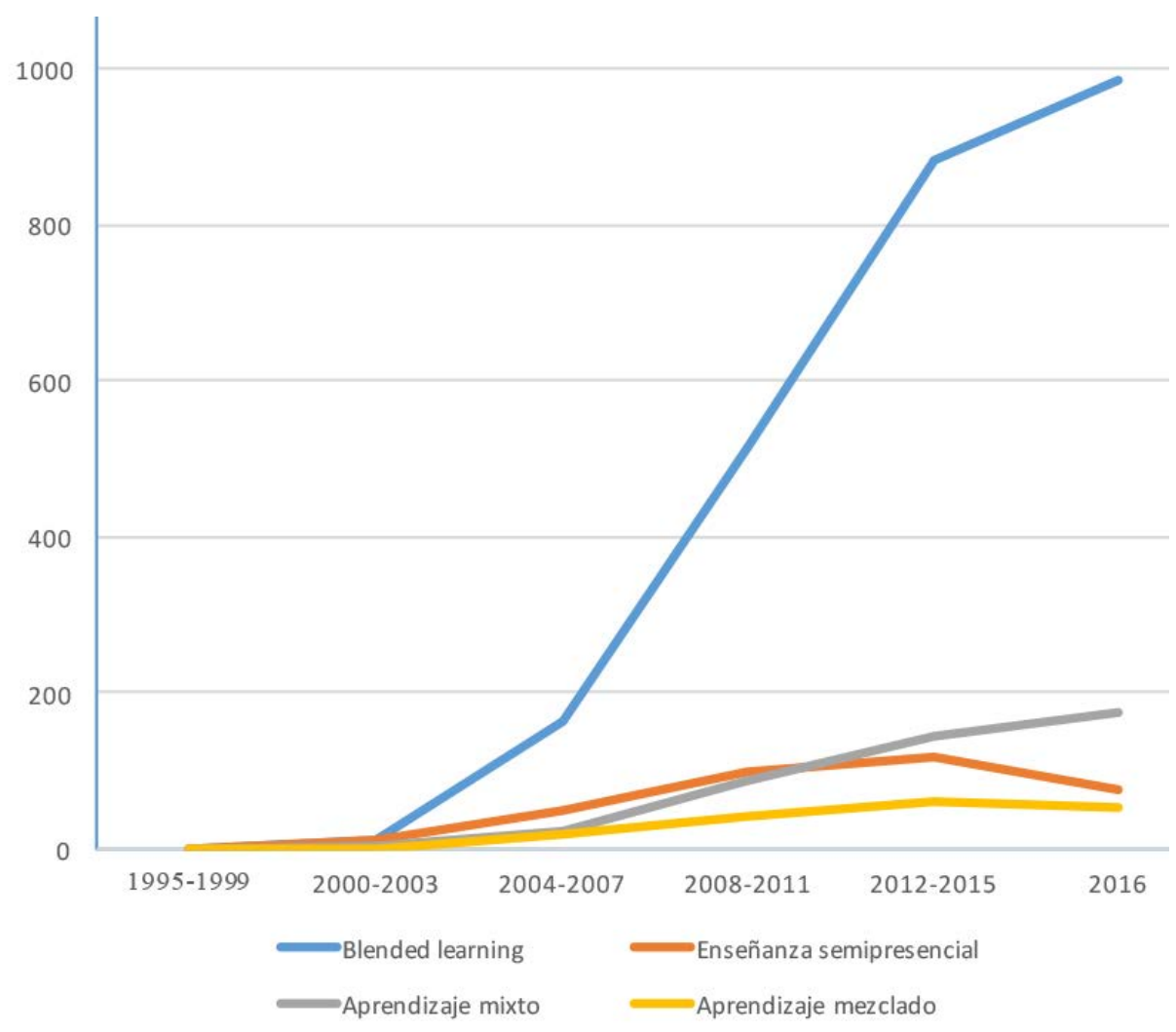

Muchos autores han resaltado en los últimos años la "efectividad" del blended learning frente a las clases presenciales (Niekerk y Webb, 2016), aunque aparecen factores como la gestión del trabajo o las actitudes de los estudiantes que influyen y predicen el éxito de estos diseños “mezclados” (Kintu y Zhu, 2016).

Sin embargo, la investigación hoy se plantea interrogantes más profundos que la mera comparativa entre el modelo tradicional y el BL. Se busca reflexionar sobre cuestiones de notable preocupación en las nuevas formas de enseñar y aprender:

- ¿QQué competencias previas requiere del estudiante para trabajar en BL?

- ¿Qué métodos docentes pueden aplicarse en los diseños BL?

- ¿Qué dispositivos, materiales y tecnologías son más efectivos?

- ¿En qué áreas y niveles educativos se están aplicando con éxito?

- ¿Cómo influyen e interactúan las características individuales de los alumnos con los diseños BL? 
- ¿Facilitan estos nuevos sistemas el desarrollo más intenso de competencias transversales?

- ¿Qué piensan los estudiantes? ¿Y los gestores que deben implementarlo?

Una revisión de las publicaciones más recientes sobre estos contenidos nos puede proporcionar las respuestas. Pero todavía podríamos ir un paso más: ¿Disponemos ya de trabajos de investigación, sólidos y consistentes, que puedan alumbrar la práctica educativa?, ¿̇hay ya ejemplos de buenas prácticas, susceptibles de generalización a otros entornos y momentos? Responder a estas preguntas marca nuestro objetivo: Construir una visión amplia y fundamentada en las principales investigaciones y autores de la literatura científica de cómo se está implementando hoy el blended learning, de manera que genere buenas prácticas en la aplicación de estos diseños.

\section{METODOLOGÍA}

Este trabajo presenta una panorámica de las principales tendencias de investigación sobre blended learning, a través de un análisis bibliográfico actualizado sobre los principales autores y estudios más actuales recogidos en las principales bases de datos de la literatura científica, con unas notas sobre prospectivas de futuro.

Para el análisis bibliográfico, se realizó una búsqueda de trabajos académicos publicados en la principal referencia de excelencia en literatura científica: la $W e b$ of Science de Clarivate Analytics. La búsqueda se basó en el título o en el tema del término blended learning:

- Contiene en el título OR en el tema: blended learning.

- Contiene en el tema "semipresencial" OR "aprendizaje mixto".

El resultado, a inicios de 2017, fueron 7.034 artículos a partir de todas las bases de datos que se manejan. Sin embargo, dado que este trabajo se centraba en la discriminación de estudios de máxima actualidad, se limitó la fecha de publicación a los años 2015 y 2016, reduciéndose el número de resultados a 1.799 registros. Fue necesaria una nueva acotación ya que,en una primera revisión sobre una pequeña muestra de esta selección, se reflejó la inclusión de textos vinculados a otros campos que se alejaban del interés de nuestro estudio. Se limitó de nuevo la sección a dos campos: Education y Educational research.

El número de artículos había quedado limitado a 1.133 registros. Se escogieron los 119 que la Wos colocaba en primer lugar, esto es, los 119 más citados en el año 2016. A partir de esa selección, se comenzó la lectura de los textos encontrando que 36 de ellos no se correspondían con el objeto de estudio. El motivo, por el que esos artículos aparecían en la selección de WoS, suele ser la inclusión por separado de los términos blended y learning como, por ejemplo, "employed a blended approach 
to form an extensive assessment... suitability of data-driven learning”. Así pues, la muestra quedó reducida a los 83 artículos más relevantes según WoS, publicados en el año 2016, y que trataban directamente el blended learning, bien con esa expresión o alguna de las otras indicadas en la búsqueda.

Cada artículo se analizó respondiendo a las preguntas indicadas anteriormente, y agrupando las respuestas por categorías lógicas. Por ejemplo, en relación a la pregunta 1 se obtuvieron 4 agrupaciones de referencias al tema de las competencias, que fueron:

- Búsqueda de información.

- Competencia digital.

- SRL (self-regulated learning).

- Valores / Educación en valores.

En relación a la segunda pregunta, métodos docentes, encontramos seis categorías:

- Estrategias de profesores y métodos docentes.

- Evaluación.

- Trabajo en grupo (y redes sociales).

- Proporción presencialidad-virtualidad.

- Clases tradicionales vs. Enseñanza mixta.

- Tutoría digital.

De modo similar se hizo con las otras preguntas. Los artículos y sus referencias eran agrupados en categorías creadas a partir de los mismos textos, por ser el método más adecuado para este tipo de análisis cualitativo y reflexivo.

\section{INVESTIGACIONES SOBRE BLENDED LEARNING}

A partir del proceso de revisión sistemática de la literatura científica seleccionada, hemos podido constatar, en primer lugar, que el término blended learning ha sido implementado -y también ha sido sometido- a una amplia evaluación a través de miles de investigaciones en todo el mundo. Solo centrándonos en artículos recogidos en la Web of Science encontramos más de 1.000 artículos académicos en los dos últimos años; en cambio, en el repositorio global de Google Scholar el término blended learning genera la impresionante cifra de 94.400 resultados.

En esta revisión, es en Estados Unidos donde aparece un mayor número de trabajos; sin embargo, los mismos se distribuyen también por todos los países del mundo. España destaca por un elevado número de contribuciones, en función de nuestro peso en la literatura científica. También Australia destaca por sus 
aportaciones relativas. Las investigaciones provienen de Asia, África, Latinoamérica, etc. en donde el BL, frente a los países llamados del "primer mundo", se presenta como una oportunidad para democratizar el conocimiento.

\section{La investigación sobre BL}

La investigación sobre blended learning ha tratado numerosas temáticas, adaptándose en esta década de existencia a las tendencias más actuales. La minería de datos se ha aplicado a las interacciones asíncronas en entornos BL (Borgobello y Roselli, 2016), encontrando un gran valor como predictor de problemas latentes (Kim y otros, 2016).

\section{Características individuales y diseño $B L$}

Recordando los viejos diseños ATI en los que se estudiaban las interacciones entre los medios y las características individuales, también se han analizado cómo esas diferencias entre estudiantes afectaban al éxito de diseños de BL. Se ha estudiado también la llamada "procrastinación", o tendencia a posponer tareas hasta el último momento (Yamada y otros, 2016), las percepciones y emociones (Jeong, González-Gómez y Cañada-Cañada, 2016), la motivación y los estilos de aprendizaje (Fernández Sánchez, 2011). Ellis y Bliuc (2016) sugieren que el modo cómo los estudiantes se aproximan al aprendizaje es clave en la calidad de los resultados en BL.

En una macroinvestigación que recogió a 111.256 estudiantes de 151 cursos, Rienties y Toetenel (2016) compararon los diseños de enseñanza con las conductas de aprendizaje, encontrando un valor predictivo en el modo en cómo se diseña la formación en relación a la conducta de los estudiantes en el entorno virtual y el rendimiento académico. Otros autores, como Borgobello y Roselli (2016), han asociado el resultado académico con algunas características de la interacción virtual en cursos de BL. Concretamente, parece que el uso de recursos multimedia influye en la percepción que tienen los alumnos de la utilidad y facilidad de un curso, mientras que la interactividad solo influye en la percepción de su utilidad (Cigdem y Ozturk, 2016). También se han encontrado niveles significativos de correlación entre el nivel de altruismo y las variables socioeconómicas de estudiantes adultos y la participación y el nivel de interacción (Diep y otros, 2016).

\section{Blended learning frente a otros diseños}

Otros autores han prestado más atención al diseño docente, representado a modo de ejemplo por el flipped classroom, que a la organización de los recursos. Ambos son diseños formativos y difieren, por ejemplo, en los recursos que incorporan (Zhang, 
Dang y Amer, 2016). Margulieux, McCracken y Catrambone (2016) los señalan como dos diseños más diferenciados del BL y FC: hybrid e inverted courses.

Una investigación reciente (Jeong, González-Gómez y Cañada-Cañada, 2016) apunta que el diseño FC proporciona mejores resultados, o cuando menos así lo perciben los estudiantes; sin embargo, son otros muchos estudios los que aportan y muestran resultados prometedores en el BL (Niekerk y Webb, 2016).

Grant (2016) compara cursos de idiomas BL y otros totalmente en línea, encontrando que, en los primeros, los estudiantes muestran más motivación y actitudes más favorables cuando utilizan laboratorios de ordenadores que aquellos que son totalmente virtuales.

La percepción más positiva de los estudiantes hacia los cursos BL no siempre refleja una mejora en los resultados de aprendizaje (Sajid y otros, 2016), pero incluso en esos casos, se encuentran porcentajes de estudiantes entre el 25 y el 30\% que rechazan los materiales en línea (Raymond y otros, 2016).

Algunos estudios han encontrado una mejora en la ratio de aprobados, comparando diseños BL con diseños eLearning con soporte multimedia, influyendo la dimensión emocional (Chen, Zhang y Yu, 2016).

\section{Áreas y materias en las que se aplican diseños BL}

La enseñanza semipresencial ha sido aplicada en, prácticamente, todas las áreas y niveles educativos. Se ha mostrado como un medio exitoso en temas de alfabetización y desarrollo de competencias matemáticas en escuelas de adultos (Windisch, 2016).

En biblioteconomía, se ha utilizado en la enseñanza del Inglés, analizando la relación entre los resultados de aprendizaje y diferencias de género o factores afectivos, tanto en ambientes BL como tradicionales. Chen, Zhang y Yu (2016) trabajaron sobre una muestra de 1.000 alumnos chinos y apuntan que no hallaron diferencias causadas por el género. En Educación Especial, los diseños de BL plantean interesantes posibilidades para enseñar a leer y escribir a estudiantes con síndrome de Down (Tangarife, Blanco y Díaz, 2016). En Ingeniería, el BL ha sido aplicado frecuentemente (Ellis, Pardo y Han, 2016; García-Beltrán y otros, 2016), incorporando a las clases y prácticas presenciales nuevas actividades a distancia (Corovic, Mahnic-Kalamiza y Miklavcic, 2016).

\section{Enseñanza de idiomas}

El BL se ha empleado también para potenciar las destrezas lingüísticas y la autonomía del aprendizaje en estudiantes universitarios, en estudios clásicos, comparando con el método tradicional (Banditvilai, 2016). En estas condiciones, el soporte del profesor es percibido como un elemento que influye directamente en los estudiantes (Fryer y Bovee, 2016). Es interesante contrastar cómo las creencias 
sobre el esfuerzo que requiere una tarea, influye en el valor que se le asigna y en el cumplimiento de las mismas.

También se ha aplicado a estudiantes de Primaria procedentes de diferentes países. En esta ocasión, los estudiantes expresaron su preferencia por actividades relacionadas con destrezas visuales y auditivas (García, 2016), destacando el incremento de los niveles de autonomía, dimensión que analizaremos posteriormente.

\section{Formación de profesores}

El BL ha sido especialmente aplicado a la formación del profesorado, por ejemplo, a futuros profesores de Educación Especial (Santos, Moriya y Schluenzen, 2016), o en materias relacionadas con la Psicología (Mauri, Ginesta y Rochera, 2016), señalándose la importancia de un adecuado feedback con los estudiantes.

En China se diseñaron cursos de BL para desarrollar las competencias digitales de profesores en ejercicio, con un elevado efecto motivador (Wu y otros, 2016). En profesores de Primaria y Secundaria también ha sido utilizado para fomentar el uso de las TIC, especialmente, para combatir la escasa cultura de trabajo colaborativo (González, Martín y Arriba, 2016).

\section{Ciencias de la Salud}

También en Medicina y Enfermería ha sido muy utilizado el BL (River y otros, 2016). Por ejemplo, se ha recurrido a las herramientas colaborativas en estudiantes de Odontología (Marei y Al-Khalifa, 2016), o para preparar los exámenes en estudiantes de Medicina (Lupi, Ward-Peterson y Chang, 2016). Se han encontrado evidencias de que el BL ha ayudado positivamente al desarrollo de destrezas tanto médicas (Hurst, 2016), como enfermeras (Swift, Efstathiou y Lameu, 2016).

Además de los foros, los vídeos han sido otro recurso muy apreciado en las implementaciones BL en Fisioterapia (Nicklen y otros, 2016) o Enfermería (Forbes y otros, 2016), utilizándose para formar en el cálculo de las dosis de medicamentos (Feizalahzadeh y otros, 2016) o recomendaciones nutricionales (Struempler, Parmer y Funderburk, 2016).

\section{Desarrollo de competencias transversales}

El BL ha encontrado un papel especial en el desarrollo de competencias transversales, constatando que el desarrollo de esas competencias aparece ligado a las características técnicas propias del $\mathrm{BL}$, al menos en sus presentaciones más difundidas. Así, se ha encontrado una relación estrecha entre el modo en cómo se aproximan los estudiantes a la búsqueda de información y al uso de tecnologías para la comunicación en línea (Ellis y Bliuc, 2016). 


\section{Competencia digital}

La competencia digital, o las habilidades tecnológicas, han sido estudiadas como factor de influencia y como factor influido en los entornos de blended learning (Willem, Aiello y Bartolomé, 2007). Así, la competencia tecnológica, junto al método utilizado y la motivación del estudiante, influyen en la intención de aprender en entornos de BL (Zhang, Dang y Amer, 2016).

De modo similar, ha sido analizada la introducción de las tecnologías y su aceptación en relación a los profesores (Kumar y Daniel, 2016). La introducción de las tecnologías ha sido estudiada en detalle (River y otros, 2016), encontrándose frecuentemente carencias en el dominio técnico de las mismas por parte de estudiantes y profesores (Henderson, Dalton y Cartmel, 2016).

De estos y otros trabajos, se deduce la existencia de subgrupos con diferencias cualitativas en el modo en cómo se acercan a la investigación y al uso de tecnologías en línea, afectando a la efectividad del BL (Ellis, Pardo y Han, 2016).

\section{Aprendizaje autorregulado}

Los sistemas de blended learning parecen potenciar también el aprendizaje autorregulado (SRL) (Whiteside, Dikkers y Lewis, 2016). Los datos sugieren que el BL puede promover la autonomía y el SRL potenciar la actividad de búsqueda y relación, y ayudar a los estudiantes a sentirse preparados para ir a la Universidad.

Se ha estudiado la relación entre el SRL y las conductas de aprendizaje en un entorno de BL (Yamada y otros, 2016), encontrándose que algunas destrezas específicas, como las prácticas en línea, benefician directamente la autonomía del estudiante, así como otras destrezas específicas de aprendizaje (Banditvilai, 2016).

Los resultados apuntan también hacia una progresión en el nivel de autonomía de los alumnos, en su trabajo con la plataforma, según avanzan en edad y curso de adscripción y, en líneas generales, su satisfacción con la modalidad de enseñanza semipresencial (García-Martínez, 2016). Esta competencia actúa como origen y como resultado. Así, estudiando la presencia de la competencia del aprendizaje autorregulado entre los estudiantes universitarios de un curso de BL, se encontró que ésta y el autocontrol eran predictivos del éxito académico ( $\mathrm{Zhu}, \mathrm{Au}$ y Yates, 2016). También ha sido analizado el SRL como covariante diferenciadora generadora de resultados positivos en estudios de género en relación al BL (Basol y Balgalmis, 2016).

\section{Otras competencias}

El BL se ha relacionado con otras competencias, por ejemplo, el trabajo en grupo en entornos de trabajo colaborativo (Fernández-Sánchez, 2011). Se ha estudiado la 
introducción en programas de educación en valores en Cuba (Pérezy otros, 2016). En la misma línea, un sistema de juegos en Red en diez escenarios fue utilizado para la educación moral en China, aunque se mostró una caída de interés con el transcurso del tiempo (Hong y otros, 2016).

\section{METODOLOGÍA DE LA DOCENCIA}

\section{Estrategias de los profesores y métodos docentes}

A pesar de las numerosas investigaciones sobre el BL, existen pocos trabajos centrados en lo que sucede en el aula. Sin embargo, se han estudiado las estrategias de los profesores para unificar los componentes presencial y en línea para el éxito de los sujetos. La combinación de clases presenciales, junto con actividades virtuales sincrónicas y con actividades en línea no sincrónicas, se ha mostrado como capaz de incrementar el rendimiento y las interacciones entre los estudiantes (Cardak y Selvi, 2016). En estudios de Medicina, el BL parece facilitar la flexibilidad y la autonomía en el proceso de aprendizaje de los estudiantes, aparte de desarrollar competencias transversales como ya se ha indicado (Trujillo y otros, 2016).

Entre las metodologías que se han utilizado, destaca el aprendizaje basado en problemas (PBL) (Thomas, Duddu y Gater, 2016). Algunos autores han señalado que los diseños de PBL están aplicándose cada vez más en entornos de BL y también totalmente en línea, para la búsqueda de información (Spector y otros, 2016).

En un meta-análisis sobre 755 investigaciones en el período 2000-15, el diseño más exitoso fue un programa de BL que incluía actividades eLearning y presentaciones con el uso de transparencias (tipo PowerPoint) (Harkanen y otros, 2016). Cabe mencionar que al menos fue un curso interactivo en línea.

\section{Niveles docentes}

Los diseños BL han sido aplicados especialmente en la Universidad (Ellis y Bliuc, 2016), pero también se han dado ejemplos en Primaria (González, Martín y Arriba, 2016) y, sobre todo, en Educación Secundaria (Whiteside, Dikkers y Lewis, 2016), en particular, con un diseño de flipped classroom, por ejemplo en cursos de Ciencias (Jeong, González-Gómez y Cañada-Cañada, 2016) o sobre temas culturales (Garcia, 2016). También la formación ocupacional en la empresa ha recurrido al BL (Aguaded, 2008).

Mención especial merece el uso del BL en la Universidad (Maraver, Mojarro y Aguaded, 2014; Fernández-Sánchez, 2011; Bartolomé, 2008; Guerra, González y García-Ruiz, 2010), donde los vídeos educativos han sido estudiados con un diseño en el que eran una parte importante del diseño (Brame, 2016), bien con estudiantes de primer y segundo curso (Yamada y otros, 2016), o como soporte para clases 
especialmente numerosas, como en estudios de Enfermería (River y otros, 2016) ya indicados.

Vuelve a aparecer aquí el uso de los diseños BL para el desarrollo de destrezas digitales en estudiantes universitarios (Vivas, Andrés y Goméz, 2016), como veremos a continuación.

\section{Evaluación y tutorías}

La introducción del BL, por lo que se refiere a los procesos de medición y evaluación, se ha orientado, en gran medida, al uso de pruebas y tests en línea, en especial, en la educación superior (García-Beltrán y otros, 2016). Con ayuda de Moodle, un curso de Física universitario preparó un banco de 1.000 ítems que permitía generar pruebas de 10 ítems a lo largo del semestre. Se encontró una correlación positiva entre los resultados que se obtenían a través de dichos exámenes en línea y el examen final (Feizalahzadeh y otros, 2016).

Algunos estudios han analizado este tipo de pruebas con otras variables como género o competencia de autorregulación de aprendizaje (Basol y Balgalmis, 2016).

El uso de tests en línea ha llevado a desarrollar diferentes sistemas para limitar las conductas deshonestas, mezclando herramientas tecnológicas, mediciones psicológicas y técnicas sociales para convencer a los estudiantes de que no deben copiar (Sullivan, 2016).

En una línea totalmente diferente, se ha sugerido el uso de tutorías digitales en cursos presenciales (Ungerer, 2016). Alternativamente, la introducción de tutorías personales cara a cara parece enriquecer los diseños totalmente en línea (Morton y otros, 2016).

\section{Aprender en Red}

Desde que Siemens y Downes desarrollaron sus ideas sobre conectivismo y aprendizaje en Red, se ha incrementado la implementación de redes sociales (y comunidades de aprendizaje) en los diseños docentes y, entre ellos, en los entornos de BL (Henderson, Dalton y Cartmel, 2016). El aprendizaje en grupo y la "peeragogy" también han sido trabajados en entornos de BL (River y otros, 2016). En algunos casos, se ha combinado el Social Networking Service (SNS) con clases tradicionales para mejorar los aprendizajes (Matsumoto y Kojima, 2016).

El entusiasmo de mezclar tecnologías con TBL (aprendizaje en grupo) puede no estar tan bien fundamentado como se pensaba. Pero falta más investigación, prestando especial atención a las barreras para el uso de las tecnologías por los estudiantes (River y otros, 2016). Se ha encontrado una relación moderadamente significativa entre la percepción del aprendizaje percibido y la participación en redes sociales, mientras que el aprendizaje en equipo, las destrezas de comunicación social 
y la presencia del instructor no parecen estar correlacionadas. Sin embargo, el efecto que relaciona la presencia social de los pares y el aprendizaje es el resultado más interesante (Turel, 2016). El BL genera un importante impacto, incrementando la interacción social y el aprendizaje en grupo.

Se han utilizado todo tipo de plataformas. En estudiantes universitarios se generó una comunidad Facebook, en un curso de BL, para estudiar la relación entre conductas de conocimiento compartido, autoeficacia académica y sentido de comunidad (Yilmaz, 2016).

Mientras algunos autores se han centrado en analizar los problemas de participación (Cardak y Selvi, 2016), otros han encontrado como primer predictor de rendimiento académico el tiempo dedicado a actividades de comunicación (Rienties y Toetenel, 2016). Otro estudio muestra que los alumnos con un uso medio de los entornos de participación en línea obtienen mejores resultados (Cavanaugh, Hargis y Mayberry, 2016).

\section{Ratio de virtualidad y/o presencialidad}

En este amplio abanico de propuestas metodológicas no podían faltar estudios sobre la ratio de virtualidad en los cursos de blended learning. Comparándolo con un curso tradicional totalmente presencial, una propuesta con solo tres días por semana de asistencia a la universidad resultó especialmente atractivo para los estudiantes que estaban incluso dispuestos a pagar más por esa propuesta con menos clase e idéntico contenido (Nicklen y otros, 2016). Hay que tener en cuenta que este estudio se realizó en Australia, y que los participantes analizaron los ahorros en términos de costos de transporte y tiempo empleado. Sería interesante un estudio similar en ciudades en las que los estudiantes no pueden residir en la proximidad del campus.

Algunas propuestas han sido más radicales, reduciendo la presencialidad a 1a clase semanal, estando la mayoría del contenido distribuido mediante recursos en línea (Green y Whitburn, 2016). Naturalmente esta propuesta rompe totalmente, desde el punto de vista metodológico, con la coexistencia de clases magistrales tradicionales. Este tipo de modelos tiene un especial interés para situaciones especiales como, por ejemplo, para profesores en grupos especiales de inclusión, que realizan una formación en línea con actividades presenciales los sábados (Maldonado, 2016).

\section{MATERIALES Y TECNOLOGÍAS}

River y otros (2016) han realizado una profunda revisión, observando que, en el BL, se utilizan múltiples tecnologías, destacando los recursos para el aprendizaje interactivo y las herramientas en Red. En nuestro caso, hemos encontrado dos grandes grupos: los recursos para la comunicación en línea y los recursos audiovisuales, 
aunque los estudios han trabajado también sobre los diferentes recursos propios del LMS (Tuapawa, 2016).

Otros materiales analizados por diversos autores han sido los portafolios (Porter, Kleve y Palermo, 2016), los laboratorios en línea y presenciales (Burchett y otros, 2016) y los mundos virtuales (Pellas y Boumpa, 2016).

Finalmente, ha sido objeto de estudio el diseño y evaluación de materiales y recursos (Pickering y Joynes, 2016), y la importancia de adecuarlos a los principios pedagógicos (Gawlik-Kobylinska, Trochowska y Maciejewski, 2016).

\section{Foros y otras herramientas en línea}

Las interacciones entre estudiantes, la centralidad y densidad de las redes (Ergun y Usluel, 2016), el sentimiento de pertenencia y las normas de reciprocidad, aparecen como predictores de la participación en línea (Diep y otros, 2016).

Marei y Al-Khalifa (2016) han estudiado en detalle cómo diferentes técnicas, en los foros en línea, afectan al funcionamiento de un curso de BL. Green y Whitburn (2016) también analizaron los foros, pero comparando su uso en cursos presenciales y semipresenciales, no encontrando diferencias entre ambos.

\section{Audiovisual}

Los vídeos han adquirido una gran importancia en los entornos de BL. Así en ciencias como en la Enfermería (Forbes y otros, 2016), utilizando diseños tipo flipped classroom (Brame, 2016); en cambio, en Medicina percibieron muy positivos los vídeos explicativos visionados antes de la clase (Liebert y otros, 2016). También un recurso destacable son los podcast de vídeo (vodcast), que permitieron a estudiantes de Fisioterapia practicar repetidamente después de las clases (Hurst, 2016). La presentación de los materiales en vídeo también se ha realizado mediante videoblogs, generando reacciones muy positivas en estudiantes universitarios (Liu, 2016).

Los vídeos se presentan como la primera fuente de recursos en un curso de BL en estudiantes de Medicina en Stanford (Evans y otros, 2016). Un formato valorado significativamente como mejor que otros es el que sigue Khan Academy (Liebert y otros, 2016). Otros estudios destacan los vídeos que presentan sincronizados a un profesor y unas transparencias tipo PowerPoint o el uso de videoanotaciones en línea (Monedero, Cebrián y Desennes, 2015; Thomas, Antonenko y Davis, 2016).

El audiovisual no se limita al uso de programas grabados en vídeo. Comienza a aparecer un nuevo concepto de semipresencialidad totalmente en línea, en el que las sesiones presenciales son sustituidas por videoconferencias y webinars (Szeto y Cheng, 2016), aunque no se trata de una equivalencia total, sino que se generan nuevos problemas de adaptación emocional y práctica. 


\section{GESTORES Y ESTUDIANTES, ACTORES IMPLICADOS EN EL PROCESO}

Un tema de notable interés, sobre el que aún existen pocas investigaciones, es lo que piensan los responsables universitarios sobre el BL. Ciabocchi, Ginsberg y Picciano (2016) aplicaron un cuestionario a una muestra amplia de miembros de la American Association of University Professors (AAUP), realizando un seguimiento posterior a un subgrupo reducido. Los resultados son muy interesantes, notando la pervivencia de unos tópicos que sitúan el BL como el entorno más deseable por detrás de la enseñanza tradicional, y por delante del eLearning o directamente los MOOC. No parece ser objeto de preocupación la calidad de los resultados en los cursos de BL pero sí el esfuerzo que requieren.

Porter y Graham (2016) han estudiado los mecanismos de adopción institucional del formato BL. Apuntan tres etapas diferenciadas: exploración, adopción temprana e implementación madura, estudiando si la estructura y los procesos de toma de decisiones de la institución facilita o impide esa adopción, y destacando factores como una suficiente infraestructura, un soporte tecnológico y pedagógico y el propósito de la institución, como claves de éxito. No conviene olvidar que, en muchos casos, la introducción del BL responde a planteamientos económicos (Aguaded y Fandos, 2008).

También se han encontrado estudios que han tratado de recoger las reacciones de los estudiantes a la implementación de cursos de BL (Hinojo, Aznar y Cáceres, 2009), en especial, sobre sus preferencias sobre diferentes actividades y modalidades (García-Martínez, 2016).

\section{DISCUSIÓN Y CONCLUSIÓN}

El blended learning está suponiendo no solo un nuevo término, sino también un nuevo concepto de entender los procesos de enseñanza-aprendizaje. No solo se han trastocado las coordenadas del espacio y del tiempo, sino que las metodologías didácticas (de enseñar y aprender) están transformándose a pasos agigantados en los dos últimos decenios. La popularización de la conectividad en todos los entornos sociales (edades, regiones, clases sociales) y para todo tipo de actividades (ocio, trabajo, educación, etc.) nos permite hoy entender que el BL tiene muchas potencialidades de implementación.

En este trabajo, hemos explicitado algunos de los principales estudios que la literatura científica de mayor prestigio internacional (indexada en el Journal Citation Reports, de Clarivate Analytics) ha recogido en los dos últimos años, constatándose, en primer lugar, la multiplicación de experiencias en todas las regiones del mundo, pero especialmente en los países con mayor presencia de las tecnologías en los entonos educativos. Pero también en países en desarrollo donde el BL se ve como una nueva oportunidad de avanzar en una mayor y mejor calidad 
en todas las capas poblacionales. En este sentido, se han evidenciado las líneas de investigación focalizadas en el BL, que destacan la pertinencia de este diseño para la enseñanza de determinadas materias y el desarrollo de competencias clave. Por otro lado, los estudios analizados muestran el potencial del BL en diversas propuestas metodológicas que propician el interés creciente por esta modalidad educativa.

El blended learning, tal como hemos reflejado en los resultados presentados, se ha comparado con otras modalidades de enseñanza-aprendizaje y se ha estudiado a fondo en los diferentes niveles del sistema educativo, así como en diferentes áreas curriculares; todo ello para dar respuesta a las preguntas de investigación planteadas. Especialmente, ha tenido un fuerte impacto en la enseñanza superior ya que las Universidades han visto una nueva oportunidad, junto a los MOOC, de abrir nuevos espacios al saber.

Blended learning, eLearning, mobile learning, flipped classroom, gamificación, realidad aumentada, MOOC, etc., son nuevos términos que se abren a los nuevos horizontes educativos, y solo en la medida que los estudiemos en profundidad y partamos de buenas prácticas, podremos rentabilizar las infinitas posibilidades, aun sin explotar, que los nuevos medios interactivos y digitales nos ponen ya en nuestras manos. La enseñanza del futuro puede ser ya presente.

\section{REFERENCIAS BIBLIOGRÁFICAS}

Aguaded, I., y Fandos, M. (2008). Web 2 (y 3.0) desde una óptica empresarial. Edutec, 26. doi: https://doi.org/10.21556/ edutec.2008.26.467

Aguaded, I., y Fandos, M. (2008). Blended Learning: The Key to Success in a Training Company. ITDL, International Journal of Instructional Technology and Distance Learning, 5(8), 1-10.

Aguaded, I., y Cabero, J. (2013). Tecnologías y medios para la educación en la $e$-sociedad. Madrid: Alianza.

Banditvilai, C. (2016). Enhancing Students' Language Skills through Blended Learning. Electronic Journal of E-Learning, 14(3), 223-232.

Bartolomé, A. R. (2004). Blended Learning. Conceptos básicos. Pixel-Bit, 23, 7-20.

Bartolomé, A. R. (2008). Entornos de aprendizaje mixto en Educación Superior. RIED. Revista Iberoamericana de Educación a Distancia, 11(1), 15-51.
Basol, G., y Balgalmis, E. (2016). A multivariate investigation of gender differences in the number of online tests received-checking for perceived self-regulation. Computers in Human Behavior, 58, 388-397. doi: https://doi. org/10.1016/j.chb.2016.01.010

Borgobello, A., y Roselli, N. D. (2016). Rendimiento académico e interacción sociocognitiva de estudiantes en un entorno virtual. Educação e Pesquisa, 42(2), 359-374. doi: https://doi. org/10.1590/S1517-9702201606143478

Brame, C. J. (2016). Effective Educational Videos: Principles and Guidelines for Maximizing Student Learning from Video Content. CBE-Life Sciences Education, 15(4). doi: https://doi.org/10.1187/ cbe.16-03-0125

Burchett, S., Hayes, J., Pfaff, A., Satterfield, E. T., Skyles, A., y Woelk, K. (2016). Piloting Blended Strategies To Resolve Laboratory Capacity Issues in a First- 
Semester General Chemistry Course. Journal of Chemical Education, 93(7), 1217-1222. doi: https://doi.org/10.1021/ acs.jchemed.6bo0078

Cardak, C. S., y Selvi, K. (2016). Increasing teacher candidates' ways of interaction and levels of learning through action research in a blended course. Computers in Human Behavior, 61, 488-506. doi: https://doi. org/10.1016/j.chb.2016.03.055

Cavanaugh, C., Hargis, J., y Mayberry, J. (2016). Participation in the Virtual Environment of Blended College Courses: An Activity Study of Student Performance. International Review of Research in Open and Distributed Learning, 17(3), 263-275.

Ciabocchi, E., Ginsberg, A., y Picciano, A. (2016). A Study of Faculty Governance Leaders' Perceptions of Online and Blended Learning. Online Learning, 2O(3), 52-73.

Cigdem, H., y Ozturk, M. (2016). Factors Affecting Students' Behavioral Intention to Use LMS at a Turkish Post-Secondary Vocational School. International Review of Research in Open and Distributed Learning, 17(3), 276-295.

Corovic, S., Mahnic-Kalamiza, S., y Miklavcic, D. (2016). Education on electrical phenomena involved in electroporationbased therapies and treatments: a blended learning approach. Biomedical Engineering Online, 15. doi: https://doi. org/10.1186/s12938-016-0152-7

Chen W., Zhang J., y Yu, Z. (2016). Learning Outcomes and Affective Factors of Blended Learning of English for Library Science. International Journal of Information and Communication Technology Education, 12(3), 13-25. doi: https://doi. org/10.4018/IJICTE.2016070102

Diep, N. A., Cocquyt, C., Zhu, C., y Vanwing, T. (2016). Predicting adult learners' online participation: Effects of altruism, performance expectancy, and social capital. Computers \& Education, 101,
84-101. doi: https://doi.org/10.1016/j. compedu.2016.06.002

Ellis, R. A., y Bliuc, A. (2016). An exploration into first-year university students' approaches to inquiry and online learning technologies in blended environments. British Journal of Educational Technology, 47(5), 970-980. doi: https:// doi.org/10.1111/bjet.12385

Ellis, R. A., Pardo, A., y Han, F. (2016). Quality in blended learning environments - Significant differences in how students approach learning collaborations. Computers \& Education, 102, 90102. doi: https://doi.org/10.1016/j. compedu.2016.07.006

Ergun, E., y Usluel, Y. K. (2016). An Analysis of Density and Degree-Centrality According to the Social Networking Structure Formed in an Online Learning Environment. Educational Technology \& Society, 19(4), 34-46.

Evans, K. H., Thompson, A. C., O’Brien, C., Bryant, M., Basaviah, P., Prober, C., y Popat, R. A. (2016). An Innovative Blended Preclinical Curriculum in Clinical Epidemiology and Biostatistics: Impact on Student Satisfaction and Performance. Academic Medicine, 91(5), 696-700. doi: https://doi.org/10.1097/ ACM.0000000000001085

Feizalahzadeh, H., Valizadeh, S., Avari, M., y Virani, F. (2016). A Study on the Effect of Lecture and Multimedia Software on Drug Calculation and Prescription: A Systematic Review. Crescent Journal of Medical and Biological Sciences, 3(3), 7580.

Fernández-Sánchez, N. (2011). Promoción del cambio de estilos de aprendizaje y motivaciones en estudiantes de educación superior mediante actividades de trabajo colaborativo en blended learning. RIED. Revista Iberoamericana de Educación a Distancia, 14(2), 189-208. doi: https:// doi.org/10.5944/ried.2.14.795 
Forbes, H., Oprescu, F. I., Downer, T., Phillips, N. M., McTier, L., Lord, B., y Visser, I. (2016). Use of videos to support teaching and learning of clinical skills in nursing education: A review. Nurse Education Today, 42, 53-56. doi: https:// doi.org/10.1016/j.nedt.2016.04.010

Fryer, L. K., y Bovee, H. N. (2016). Supporting students' motivation for e-learning: Teachers matter on and offline. Internet and Higher Education, 3O, 21-29. doi: https://doi.org/10.1016/j. iheduc.2016.03.003

García-Martínez, S. (2016). Percepciones y valoraciones de alumnos en edad escolar sobre su experiencia de aprendizaje en un programa semipresencial de lengua y cultura españolas. RIED, 19(2), 237-262. https://doi.org/10.5944/ried.19.2.15398

García-Beltrán, A., Martínez, R., Jaén, J., y Tapia, S. (2016). Self-assessment in virtual teaching and learning environments. RED, 5O. doi: https://doi.org/10.6018/ red/50/14

Gawlik-Kobylinska, M., Trochowska, K., y Maciejewski, P. (2016). Civil-military intercultural education and training in the form of blended learning. E-Mentor, $3,24-34$.

González, M. C., Martín, S. C., y Arriba, J. M. (2016). Experiencias de trabajo colaborativo mediante Tecnologías de la Información y la Comunicación entre profesores. Revista Portuguesa de Educação, 29(1), 75-98. doi: https://doi. org/10.21814/rpe.6996

Grant, S. (2016). Peer review process completion rates and subsequent student perceptions within completely online versus blended modes of study. System, 62, 93-101. doi: https://doi.org/10.1016/j. system.2016.06:010

Green, R. A., y Whitburn, L. Y. (2016). Impact of Introduction of Blended Learning in Gross Anatomy on Student Outcomes. Anatomical Sciences Education, 9(5),
422-430. doi: https://doi.org/10.1002/ ase. 1602

Guerra, S., González, N., y García-Ruiz, R. (2010). Utilización de las TIC por el profesorado universitario como recurso didáctico [Study on the Use of ICTs as Teaching Tools by University Instructors]. Comunicar, 35, 141-148. doi: https://doi. org/10.3916/C35-2010-03-07

Harkanen, M., Voutilainen, A., Turunen, E., y Vehvilainen-Julkunen, K. (2016). Systematic review and meta-analysis of educational interventions designed to improve medication administration skills and safety of registered nurses. Nurse Education Today, 41, 36-43. doi: https:// doi.org/10.1016/j.nedt.2016.03.017

Henderson, S., Dalton, M. y Cartmel, J. (2016). Using Interprofessional Learning for Continuing Education: Development and Evaluation of the Graduate Certificate Program in Health Professional Education for Clinicians. Journal of Continuing Education in the Health Professions, 36(3), 211-217. doi: https://doi. org/10.1097/CEH.0000000000000093

Hinojo, F. J., Aznar, I., y Cáceres, M. P. (2009). Percepciones del alumno sobre el blended learning en la Universidad. Comunicar, 33, 165-174.

Hong, J., Hwang, M., Wu, N., Huang, Y., Lin, P., y Chen, Y. (2016). Integrating a moral reasoning game in a blended learning setting: effects on students' interest and performance. Interactive Learning Environments, 24(3), 572-589. doi: https://doi.org/10.1080/10494820.2014 .908926

Hurst, K. M. (2016). Using video podcasting to enhance the learning of clinical skills: A qualitative study of physiotherapy students' experiences. Nurse Education Today, 45, 206-211. doi: https://doi. org/10.1016/j.nedt.2016.08.011

Jeong, J. S., González-Gómez, D., y CañadaCañada, F. (2016). Students' Perceptions and Emotions Toward Learning in a 
Flipped General Science Classroom. Journal of Science Education and Technology, 25(5), 747-758. doi: https:// doi.org/10.1007/s10956-016-9630-8

Kim, D., Park, Y., Yoon, M., y Jo, I. (2016). Toward evidence-based learning analytics: Using proxy variables to improve asynchronous online discussion environments. Internet and Higher Education, 30, 30-43. doi: https://doi. org/10.1016/j.iheduc.2016.03.002

Kintu, M. J., y Zhu, C. (2016). Student Characteristics and Learning Outcomes in a Blended Learning Environment Intervention in a Ugandan University. Electronic Journal of e-Learning, 14(3), 181-195.

Kumar, S., y Daniel, B. K. (2016). Integration of learning technologies into teaching within Fijian Polytechnic Institutions. International Journal of Educational Technology in Higher Education, 13. doi: https://doi.org/10.1186/s41239-0160036-8

Liebert, C. A., Mazer, L., Merrell, S. B., Lin, D. T., y Lau, J. N. (2016). Student perceptions of a simulation-based flipped classroom for the surgery clerkship: A mixed-methods study. Surgery, 160(3), 591-598. doi: https://doi.org/10.1016/j. surg.2016.03.034

Liu, M. (2016). Blending a class video blog to optimize student learning, outcomes in higher education. Internet and Higher Education, 30, 44-53. doi: https://doi. org/10.1016/j.iheduc.2016.03.001

Lupi, C., Ward-Peterson, M., y Chang, W. (2016). Advancing non-directive pregnancy options counseling skills: A pilot Study on the use of blended learning with an online module and simulation. Contraception, 94(4), 348352. doi: https://doi.org/10.1016/j. contraception.2016.03.005

Maldonado Molina Pagnez, K. S. (2016). Training of Teachers for Acting in the School Inclusion. Journal of Research in
Special Educational Needs, 16, 70-74. doi: https://doi.org/10.1111/1471-3802.12127

Maraver, P., Mojarro, A., y Aguaded, I. (2014). Social Network Analysis of a Blended Learning Experience in Higher Education. Research on Education and Media. Recuperado de http://rem. pensamultimedia.it

Marei, H. F., y Al-Khalifa, K. S. (2016). Pattern of online communication in teaching a blended oral surgery course. European Journal of Dental Education, 2O(4), 213-217. doi: https://doi. org/10.1111/eje.12163

Margulieux, L. E., McCracken, W. M., y Catrambone, R. (2016). A taxonomy to define courses that mix face-to-face and online learning. Educational Research Review, 19, 104-118. doi: https://doi. org/10.1016/j.edurev.2016.07.001

Matsumoto, S., y Kojima, H. (2016). Constructing an Interaction Support System with the Capability of Social Networking Service for a Practical Lecture and Examining its Efficient Operational Policy. International Journal of Knowledge and Systems Science, 7(2), 1-27. doi: https://doi.org/10.4018/ IJKSS.2016040101

Mauri, T., Ginesta, A., y Rochera, M. (2016). The use of feedback systems to improve collaborative text writing: A proposal for the higher education context. Innovations in Education and Teaching International, 53(4), 411-423. doi: https://doi.org/10.10 80/14703297.2014.961503

Monedero, J. J., Cebrián, D., y Desenne, P. (2015). Usability and Satisfaction in Multimedia Annotation Tools for MOOCs [Usabilidad y satisfacción en herramientas de anotaciones multimedia para MOOC]. Comunicar, 44, 55-62. doi: https://doi. org/10.3916/C44-2015-06

Morton, C. E., Saleh, S. N., Smith, S. F., Hemani, A., Ameen, A., Bennie, T. D., y Toro-Troconis, M. (2016). Blended learning: how can we optimise 
undergraduate student engagement? BMC Medical Education, 16. doi: https:// doi.org/10.1186/s12909-016-0716-Z

Nicklen, P., Rivers, G., Ooi, C., Ilic, D., Reeves, S., Walsh, K., y Maloney, S. (2016). An Approach for Calculating Student-Centered Value in Education A Link between Quality, Efficiency, and the Learning Experience in the Health Professions. Plos One, 11(9). doi: https:// doi.org/10.1371/journal.pone.0162941

Pellas, N., y Boumpa, A. (2016). Open Sim and Sloodle Integration for Preservice Foreign Language Teachers' Continuing Professional Development: A Comparative Analysis of Learning Effectiveness Using the Community of Inquiry Model. Journal of Educational Computing Research, 54(3), 407-440. doi: https:// doi.org/10.1177/0735633115615589

Pérez-Domínguez, Y., Tellez-Carralero, A., Cespedes-Labrada, T., y Lemes-Pérez, A. (2016). The Class Meeting, essential guide for achieving education through instruction in Municipal University Centers. Dilemas Contemporaneos. Educacion Política y Valores, 3(3).

Pickering, J. D., y Joynes, V. C. (2016). A holistic model for evaluating the impact of individual technology-enhanced learning resources. Medical Teacher, 38(12), 1242-1247. doi: https://doi. org/10.1080/0142159X.2016.1210112

Porter, J., Kleve, S., y Palermo, C. (2016). An exploratory study comparing two electronic portfolio approaches in undergraduate dietetic education. Nutrition \& Dietetics, 73(3), 235-240. doi: https://doi.org/10.1111/1747-0080.12210

Porter, W. W., y Graham, C. R. (2016). Institutional drivers and barriers to faculty adoption of blended learning in higher education. British Journal of Educational Technology, 47(4), 748-762. doi: https://doi.org/10.1111/bjet.12269

Raymond, A., Jacob, E., Jacob, D., y Lyons, J. (2016). Peer learning a pedagogical approach to enhance online learning: A qualitative exploration. Nurse Education Today, 44, 165-169. doi: https://doi. org/10.1016/j.nedt.2016.05.016

Rienties, B., y Toetenel, L. (2016). The impact of learning design on student behaviour, satisfaction and performance: A cross-institutional comparison across 151 modules. Computers in Human Behavior, 6o, 333-341. doi: https://doi. org/10.1016/j.chb.2016.02.074

River, J., Currie, J., Crawford, T., Betihavas, V., y Randall, S. (2016). A systematic review examining the effectiveness of blending technology with team-based learning. Nurse Education Today, 45, 185-192. doi: https://doi.org/10.1016/j. nedt.2016.08.012

Rubia, B., y Guitert, M. (2014). ¿La revolución de la enseñanza? El aprendizaje colaborativo en entornos virtuales (CSCL) [Revolution in Education: Computer Support for Collaborative Learning]. Comunicar, 42, 10-14. doi: https://doi. org/10.3916/C42-2014-a2

Sajid, M. R., Laheji, A. F., Abothenain, F., Salam, Y., AlJayar, D., y Obeidat, A. (2016). Can blended learning and the flipped classroom improve student learning and satisfaction in Saudi Arabia? International Journal of Medical Education, 7, 281-285. doi: https://doi. org/10.5116/ijme.57a7.83d4

Salinas Ibáñez, J. (1997). Modelos mixtos de formación universitaria presencial ya distancia: el Campus Extens. Cuadernos de Documentación Multimedia, 6-7, 5564.

Santos, D. A., Moriya Schluenzen, E. T., y Schluenzen, K. J. (2016). Training For Inclusive Education and Special. Journal of Research in Special Educational. Needs, 16, 539-543. doi: https://doi. org/10.1111/1471-3802.12184

Spector, J. M., Ifenthaler, D., Sampson, D., Yang, L., Mukama, E., Warusavitarana, A., y Gibson, D. C. (2016). Technology 
Enhanced Formative Assessment for 21st Century Learning. Educational Technology \& Society, 19(3), 58-71.

Struempler, B., Parmer, S. M., y Funderburk, K. (2016). Use of Blended Learning to Improve Nutrition Knowledge in ThirdGraders. Journal of Nutrition Education and Behavior, 48(7), 510-511. doi: https:// doi.org/10.1016/j.jneb.2016.04.401

Sullivan, D. P. (2016). An Integrated Approach to Preempt Cheating on Asynchronous, Objective, Online Assessments in Graduate Business Classes. Online Learning, 2O(3), 195-209.

Swift, A., Efstathiou, N., y Lameu, P. (2016). Is LabTutor a helpful component of the blended learning approach to biosciences? Journal of Clinical Nursing, 25(17-18), 2683-2693. doi: https://doi.org/10.1111/ jocn.13175

Szeto, E., y Cheng, A. Y. N. (2016). Towards a framework of interactions in a blended synchronous learning environment: what effects are there on students' social presence experience? Interactive Learning Environments, 24(3), 487-503. doi: https://doi.org/10.1080/10494820.2 014.881391

Tangarife-Chalarca, D., Blanco-Palencia, M., y Díaz-Cabrera, G. M. (2016). Technologies and Methodologies Applied in the Teaching of Literacy to People with Down syndrome. Digital Education Review, 29, 265-282.

Thomas, A. O., Antonenko, P. D., y Davis, R. (2016). Understanding metacomprehension accuracy within video annotation systems. Computers in Human Behavior, 58, 269-277. doi: $\quad$ https://doi.org/10.1016/j. chb.2016.01.014

Thomas, G., Duddu, V., y Gater, R. (2016). Blending an e-learning package into a problem-based learning module. International Journal of Medical Education, 7, 259-260. doi: https://doi. org/10.5116/ijme.5787.617d
Trujillo-Maza, E. M., Gómez-Lozano, M. T., Cardozo Alarcon, A. C., Moreno Zuluaga, L., y Gamba Fadul, M. (2016). Blended learning supported by digital technology and competency-based medical education: A case study of the social medicine course at the Universidad de los Andes, Colombia. International Journal of Educational Technology in Higher Education, 13. doi: https://doi. org/10.1186/s41239-016-0027-9

Tuapawa, K. (2016). Interpreting Experiences of Teachers Using Online Technologies to Interact with Students in Blended Tertiary Environments. International Journal of Information and Communication Technology Education, 12(4), 76-87. doi: https://doi. org/10.4018/IJICTE.2016100107

Turel, Y. K. (2016). Relationships between students' perceived team learning experiences, team performances, and social abilities in a blended course setting. Internet and Higher Education, 31, 79-86. doi: https://doi.org/10.1016/j. iheduc.2016.07.001

Ungerer, L. M. (2016). Digital Curation as a Core Competency in Current Learning and Literacy: A Higher Education Perspective. International Review of Research in Open and Distributed Learning, 17(5), 1-27.

Vivas Urias, M. D., Andrés-Ortega, S., y Gómez-Navarro, M. (2016). Development of Digital Competencies in Online Instruction: The Case Study of the Foundation Subject for the Building Engineering Degree. Red, 49. doi: https:// doi.org/10.6018/red/49/8

Whiteside, A. L., Dikkers, A. G., y Lewis, S. (2016). More Confident Going into College: Lessons Learned from Multiple Stakeholders in a New Blended Learning Initiative. Online Learning, 20(4), 136156.

Willem, C., Aiello, M., y Bartolomé, A. R. (2007). Blended Learning and New 
Literacies. The International Journal of Technology, Knowledge \& Society, 2, 3-9. Windisch, H.C. (2016). How to motivate adults with low literacy and numeracy skills to engage and persist in learning: A Literature review of Policy Interventions. International Review of Education, 62(3), 279-297. doi: https://doi.org/10.1007/ s11159-016-9553-X

Wu, B., Hu, Y., Gu, X., y Lim, C. P. (2016). Professional Development of New Higher Education Teachers With Information and Communication Technology in Shanghai: A Kirkpatrick's Evaluation Approach. Journal of Educational Computing Research, 54(4), 531-562. doi: https:// doi.org/10.1177/0735633115621922

Yamada, M., Goda, Y., Matsuda, T., Saito, Y., Kato, H., y Miyagawa, H. (2016). How does self-regulated learning relate to active procrastination and other learning behaviors? Journal of Computing In Higher Education, 28(3), 326-343. doi: https://doi.org/10.1007/s12528-0169118-9

Yilmaz, R. (2016). Knowledge sharing behaviors in e-learning community: Exploring the role of academic selfefficacy and sense of community. Computers in Human Behavior, 63, 373-382. doi: https://doi.org/10.1016/j. chb.2016.05.055

Zhu, Y., Au, W., y Yates, G. (2016). University students' self-control and self-regulated learning in a blended course. Internet And Higher Education, 30, 54-62. doi: https:// doi.org/10.1016/j.iheduc.2016.04.001

\section{PERFIL ACADÉMICO Y PROFESIONAL DE LOS AUTORES}

Antonio Bartolomé Pina. Doctor en Filosofía y Ciencias de la Educación por la Universitat de Barcelona. Catedrático de Medios Digitales y Educación, y Director del Instituto de Investigación en Educación de la Universitat de Barcelona. Ha dirigido más de 20 proyectos internacionales $\mathrm{I}+\mathrm{D}+\mathrm{I}$ en las líneas del diseño de entornos de aprendizaje potenciado por la tecnología y de Alfabetización e inclusión digital. Es autor de 30 libros y 147 artículos. Ha impartido 230 conferencias en México, Chile, Brasil, España, Colombia, Perú, Costa Rica, Argentina, Estados Unidos de América, Bélgica, Italia, Canadá, Portugal, Guatemala y Alemania. Ha sido profesor visitante en las Universidades de Guadalajara, Monterrey, Canterbury, Federal de Santa Catalina, Católica de Chile, El Salvador, Tecnológico de Monterrey, Calgary, entre otras, además de impartir 220 cursos en universidades y centros de profesores de los países citados. http://www.lmi.ub.edu/personal/bartolome/

E-mail: abartolomepina@gmail.com

\section{DIRECCIÓN DEL AUTOR}

Ptge. De les roses, 1

o8340 Vilassar de Mar

(Barcelona), España 
Rosa García-Ruiz. Doctora en Ciencias de la Educación por la UNED (España). Profesora de la Universidad de Cantabria, adscrita al área de Didáctica y Organización Escolar, participando en Grados, Máster y Doctorado. Profesora del Máster de Comunicación y Educación Audiovisual (UHU-UNIA). Profesora del Doctorado Interuniversitario en Comunicación de las universidades de Cádiz, Huelva, Málaga y Sevilla. Editora adjunta de la Revista Comunicar y editora jefa de Alteridad: Revista de Educación. Coordinadora de la sección española de la Red Alfamed, y del grupo de investigación EDUCAMED, de la Universidad de Cantabria. Ha colaborado en varios proyectos competitivos nacionales e internacionales, y ha sido IP de varios contratos con la Administración. Ha publicado artículos, libros y capítulos de libros nacionales e internacionales. Sus principales líneas de investigación son la alfabetización mediática y digital, metodologías y recursos para la formación del profesorado.

E-mail: rosa.garcia@unican.es

\section{DIRECCIÓN DE LA AUTORA}

Facultad de Educación. Edificio Interfacultativo

Universidad de Cantabria

Avd. Los Castros s/n

39005 - Santander (Cantabria) España

Ignacio Aguaded. Catedrático de Universidad del Departamento de Educación de la Universidad de Huelva (España). Presidente del Grupo Comunicar, colectivo veterano en España en Educomunicación, y Editor de la reconocida revista científica Comunicar (indexada en JCR, Scopus, ERIH, RECYT...). Es, además, Director del Grupo de Investigación «Ágora», responsable de múltiples investigaciones nacionales e internacionales y de la dirección de numerosas tesis doctorales. Es Director del Máster Internacional Interuniversitario de Comunicación y Educación Audiovisual (UNIA/UHU) y Subdirector del Programa Interuniversitario de Comunicación (US, UMA, UCA y UHU). Premio de Mejor Investigador de la Universidad de Huelva 2015 en Ciencias Sociales. Ha sido Vicerrector de Tecnologías, Innovación y Calidad de la Universidad de Huelva durante 7 años (2005-12).

E-mail: aguaded@uhu.es

\section{DIRECCIÓN DEL AUTOR}

Facultad de Ciencias de la Educación

Universidad de Huelva

21071 Huelva (Spain) 
Fecha de recepción del artículo: 21/04/2017

Fecha de aceptación del artículo: 21/06/2017

\section{Como citar este artículo:}

Bartolomé, A., García-Ruiz, R., y Aguaded, I. (2018). Blended learning: panorama y perspectivas. RIED. Revista Iberoamericana de Educación a Distancia, 21(1), pp. 33-56. doi: http://dx.doi.org/10.5944/ried.21.1.18842 